\title{
Desfechos clínicos dos idosos hospitalizados com COVID-19
}

\author{
Clinical outcomes of elderly hospitalized with COVID-19
}

Resoluciones clínicas de los ancianos hospitalizados con COVID-19

\author{
Aline da Silva Paula \\ ORCID: https://orcid.org/0000-0001-5038-3570 \\ Universidade Federal do Paraná, Brasil \\ E-mail: silvapaula@ufpr.br \\ Karina Silveira de Almeida Hammerschmidt \\ ORCID: https://orcid.org/0000-0002-7140-3427 \\ Universidade Federal do Paraná, Brasil \\ E-mail: ksalmeidah@ufpr.br \\ Maria Helena Lenardt \\ ORCID: https://orcid.org/0000-0001-8309-4003 \\ Universidade Federal do Paraná, Brasil \\ E-mail: curitiba.helena@gmail.com \\ Neidamar Pedrini Arias Fugaça \\ ORCID: https://orcid.org/0000-0002-2112-0920 \\ Universidade Federal do Paraná, Brasil \\ E-mail: neidamar.arias@ufpr.br \\ Alcione Oliveira de Souza \\ ORCID: https://orcid.org/0000-0002-3193-3642 \\ Universidade Federal do Paraná, Brasil \\ E-mail: alcionesouza@ufpr.br \\ Luciane Lachouski \\ ORCID: https://orcid.org/0000-0003-1543-8323 \\ Universidade Federal do Paraná, Brasil \\ E-mail: lucianelachouski@ufpr.br
}

\begin{abstract}
Resumo
Estudo quantitativo documental com objetivo de analisar desfechos clínicos e características sociodemográficas e clínicas de idosos hospitalizados pelo Coronavírus (COVID-19). Foi realizada pesquisa documental, com dados do Núcleo de Epidemiologia Hospitalar sobre idosos hospitalizados com COVID-19 em 2020. Os resultados mostram que predominou o sexo masculino (50,3\%), a idade média de 71,9, e as multimorbidades (88,8\%). No prognóstico, 70,9\% receberam alta, 22,9\% óbitos e 6,1\% transferência para outros hospitais. Nas altas prevaleceram: idade média de 70,4; sexo masculino 53,3\%, uso de suporte de oxigênio (72,44\%), em que 5,5\% utilizaram ventilação mecânica, foram pronados $(79,53 \%)$, tiveram média de pressão parcial de Oxigênio (PaO2) de 102,79 \pm 47,47, D-Dímero de $2016,98 \pm 1994,94$, e o exame de tomografia com aspecto em opacidades ocorreu em 66,79\%. Nos óbitos predominaram: idade média de 76,6, sexo feminino (58,5\%), necessidade de oxigenoterapia em 97,53\% dos idosos, uso de ventilador mecânico (VM) 58,54\% e posição de prona em 60,98\%, média D-Dímero de 5022,29, exame de tomografia com aspecto em opacidades em 73,2\%. Por fim, nos desfechos alta e óbito, as morbidades prevalentes foram: HAS, diabetes Mellitus e doença cardiovascular. A idade média foi de 70,4 (alta) e 76,6 (óbito). Principal alteração na tomografia foi opacidades em vidros fosco $(73,2 \%), 97,53 \%$ utilizaram oxigênio, e 58,54\% necessitaram de intubação.

Palavras-chave: Idoso; Coronavírus; Hospitais; Dados demográficos; Enfermagem geriátrica.
\end{abstract}

\begin{abstract}
Quantitative documentary aimed to analyze clinical outcomes and sociodemographic and clinical characteristics of elderly hospitalized by Coronavirus (COVID-19). Documental research was realized, with data from Hospital Epidemiology Center on elderly people hospitalized with COVID-19 in 2020. The results show male predominated $(50.3 \%)$, mean age of 71.9 , and multimorbidities $(88.8 \%)$. In the prognosis, $70.9 \%$ were discharged $22.9 \%$ died and $6.1 \%$ were transferred to other hospitals. At discharges prevailed: mean age of 70.4; male 53.3\%, used oxygen support $(72.44 \%)$, in which $5.5 \%$ used mechanical ventilation, were prone $(79.53 \%)$, had a mean partial pressure of oxygen (PaO2) by $102.79 \pm 47.47$, D-Dimer of $2016.98 \pm 1994.94$, and the Computed Tomography (CT) scan with aspect in opacities occurred in $66.79 \%$. In deaths, predominated: mean age of 76.6 , female $(58.5 \%)$, need oxygen therapy in $97.53 \%$ of the elderly, use of mechanical ventilation (MV) $58.54 \%$ and prone position in $60.98 \%$, mean D-Dimer of 5022.29 , Computed Tomography (CT) scan with aspect in opacities in $73.2 \%$. Finally, in discharge and death outcomes the prevalent morbidities: SAH, diabetes Mellitus and cardiovascular disease. The mean age was 70.4 (discharge) and $76.7 \%$ (death), $97.53 \%$ used oxygen, and $58.54 \%$ required intubation.
\end{abstract}

Keywords: Aged; Coronavirus; Hospitals; Demographic data; Geriatric nursing. 


\begin{abstract}
Resumen
Estudio cuantitativo documental con el objetivo de analizar las resoluciones clínicas y las características sociodemográficas y clínicas de los ancianos hospitalizados por el Coronavirus (COVID-19). Fue realizada investigación documental con datos del Núcleo de Epidemiología Hospitalaria sobre los ancianos hospitalizados con COVID-19 en 2020. Los resultados muestran que predominó el sexo masculino (50,3\%), la edad media de 71,9 años y la multimorbilidad (88,8\%). En el pronóstico $70,9 \%$ fueron dados de alta, $22,9 \%$ han fallecido y el $6,1 \%$ fueron trasladados a otros hospitales. En las altas prevalecieron: edad media de 70,4 años: sexo masculino 53,5\%; uso de oxígeno de apoyo $(72,44 \%)$, en el que 5,5\% utilizó ventilación mecánica; estaban pronados (79,53\%); tenían una presión parcial media de oxígeno (PaO2) de 102,79 \pm 47,47; Dímero D de 2016,98 \pm 1994,94; y examen de tomografía con aparición de opacidades ocurrió en el 66,79\%. Los datos predominantes fueron: edad media de 76,6, sexo femenino (58,5\%), necesidad de oxigenoterapia en el $97,53 \%$ de los casos, uso de ventilador mecánico (VM) en el 58,54\% y posición de prona en el 60,98\%, media Dímero D de 5022,22, examen de tomografía con aspecto de opacidad en el $73,2 \%$. Por fin, en las resoluciones de alta y muerte las morbilidades prevalentes: Hipertensión arterial sistémica, diabetes Mellitus y enfermedades cardiovasculares. La edad media fue de 70,4 años (al alta) y 76,6 años (al fallecimiento). La principal alteración en la tomografía fue las opacidades en vidrio deslustrado (73,2\%), el 97,53\% utilizo oxígeno y el 58,54\% necesitó intubación.
\end{abstract}

Palabras clave: Anciano; Coronavirus; Hospitales; Datos demográficos; Enfermería geriátrica.

\title{
1. Introdução
}

Aliado ao contexto de envelhecimento populacional brasileiro, emergiu em 2020 a pandemia provocada pelo betacoronavírus denominado de Severe Acute Respiratory Syndrome Coronavirus-2 (Sars-Cov-2), com patologia intitulada Corona Vírus Disease-19 (COVID-19), que provoca síndroma respiratória aguda grave e ceifa milhões de vida em todo mundo (Ng \& Tilg, 2020).

No Brasil o primeiro caso confirmado de COVID-19 foi em fevereiro de 2020 com crescente e alarmante aumento do número de caso no ano recorrente. Até o dia 07 de fevereiro de 2021, o país apresentava mais de 9 milhões de casos notificados e 230 mil óbitos confirmados pela doença (Who, 2020).

A COVID-19 é caracterizada como infecção respiratória aguda potencialmente grave, a apresentação clínica tem sido de infecção respiratória cujos sintomas variam de doença leve, até pneumonia viral grave, que leva à Síndrome Respiratória Aguda Grave (SRAG), podendo ser fatal (Aquino et al., 2020).

De acordo com o Ministério da Saúde, duas situações são consideradas como caso suspeito para COVID-19: paciente com Síndrome Gripal (SG) ou com SRAG, indivíduo que apresentasse quadro respiratório agudo, a primeira situação com pelo menos dois dos seguintes sintomas: febre (aferida ou referida), tosse, dor de garganta, dor de cabeça, calafrios, coriza, distúrbios olfativos e/ou distúrbios gustativos. E, para idosos, os sintomas de síncope, irritabilidade, confusão mental, excesso de sonolência e inapetência eram considerados critérios de agravamento (Ministério da Saúde, 2020a; 2020b).

Ao analisar a distribuição dos casos do COVID-19 em 2020 e de óbitos por faixa etária, no mundo e no Brasil, verificase que existiu maior mortalidade na população adulta, contudo a maior letalidade da doença foi registrada na população idosa, sendo este grupo considerado mais suscetível aos desfechos adversos da doença (Barbosa et al., 2020).

Dados do ano de 2020, evidenciaram taxas mundiais referentes a mortalidade pelo novo Coronavírus, destacando-se os Estados Unidos como país com maior número acumulado (511.994), seguido do Brasil (254.221), México (185.257), Índia (157.051) e Reino Unido (122.939) (Ministério da Saúde, 2021).

No Brasil, 69,3\% dos óbitos ocorreram em idosos, destes $64 \%$ apresentavam pelo menos um fator de risco, principalmente Doença Crônica Não Transmissível (DCNT). Os dados epidemiológicos sobre o COVID-19 apontam que entre os idosos com 80 anos ou mais, 14,8\% dos infectados morreram, em comparação a 8,0\% dos idosos de 70 a 79 anos e 8,8\% entre os de 60 a 69 anos, revelando taxa de mortalidade 3,82 vezes maior que a média geral (Ministério da Saúde, 2021).

Neste cenário, do primeiro ano pandêmico, os idosos tornaram-se o centro da atenção em relação a COVID-19, principalmente devido ao elevado risco de letalidade, mortalidade e maior probabilidade de desenvolver a doença de forma grave. 
Esta condição está relacionada a diversidade de situações de saúde e doença que os idosos brasileiros apresentam, influenciadas por alterações na funcionalidade, multimorbidades, comorbidades, agravos e riscos para imunossupressão (Hammerschmidt \& Santana, 2020).

A Organização Mundial da Saúde (Who, 2021) considera precoce estimar os efeitos da COVID-19 a longo prazo, porém declara que os idosos, principalmente aqueles com DCNT são mais propensos a desenvolver quadros graves da doença, tendo maior chance de letalidade, em especial aqueles com 80 ou mais anos. Este achado pode ser verificado nos dados epidemiológicos brasileiros da pandemia COVID-19, em que no ano de 2020 evidenciou os idosos como grupo populacional de risco para contaminação do SARS-COV-2, demandando políticas públicas e estratégias (Oliveira et al., 2020).

A identificação de características sociodemográficas, clínicas e desfecho relacionadas ao idosos hospitalizados com COVID-19, pode contribuir para o entendimento da dinâmica da doença, além de ser crucial para o desenvolvimento de medidas de enfrentamento da pandemia e minimização dos danos nessa população específica.

É relevante analisar tais características relacionadas à população idosa, considerando os dados que envolvem desfechos clínicos, sejam eles positivos ou negativos, como informação fundamental para direcionar os cuidados de enfermagem gerontológicos desse segmento populacional. Essas informações podem oportunizar o esclarecimento de características básicas, contribuindo no desenvolvimento e implantação de cuidados e protocolos de tratamentos mais assertivos.

Nesse sentido o presente estudo teve como objetivo analisar os desfechos clínicos e as características sociodemográficas e clínicas de idosos hospitalizados pelo novo Coronavírus (Covid-19).

\section{Metodologia}

Trata-se de estudo do tipo documental realizado em um hospital privado, localizado na Cidade de Curitiba Paraná/Brasil, com dados secundários e extraídos da fonte Núcleo de Epidemiologia Hospitalar. Este estudo caracteriza-se pela exploração de dados que não receberam tratamento analítico, em que a fonte de coleta de dados foi documentos (Gil, 2008; Lakatos \& Marconi, 1991).

A análise documental foi escolhida por ser procedimento que engloba identificação, verificação e apreciação de documentos que mantêm relação com o objeto investigado (Moreira, 2009). A sua utilização contribui para a observação do processo de maturação ou de evolução de indivíduos, grupos, conceitos, conhecimentos, comportamentos, mentalidades, práticas, entre outros (Cellard, 2012).

Buscou-se esta metodologia a fim de aprofundar a investigação das características sociodemográficas, necessidades humanas afetadas e cuidados de enfermagem prescritos às pessoas idosas hospitalizadas com COVID-19 cuja análise documental caracterizada por tipologia que pode ser utilizada com possibilidade de aprofundamento teórico na temática, por meio da utilização de documentos secundários do Núcleo de Epidemiologia Hospitalar, prescrições de cuidados de enfermagem em prontuário do paciente.

O Núcleo de Epidemiologia Hospitalar é o setor responsável pelo planejamento e execução das ações de epidemiologia hospitalar, incluindo a vigilância epidemiológica das doenças de notificação compulsória e outros fatos de interesse para a saúde pública, ou seja, é o serviço do hospital responsável pela vigilância permanente dos problemas de saúde que podem pôr em risco a saúde da população (Ministério da Saúde, 2010).

A amostra foi constituída mediante período amostral, de todos os idosos hospitalizados diagnosticados com COVID-19 no período de março a dezembro de 2020, foram incluídos na amostra, os critérios de inclusão foram: idosos com idade igual e/ou maio que 60 anos, permanência hospitalar maior que 24 horas e diagnóstico positivo para COVID-19, com admissão hospitalar via pronto socorro, e os critérios de exclusão foram os idosos que vieram transferidos de outro hospital com internação 
direta na UTI ou na enfermaria mesmo que já com diagnóstico de COVID-19 (sem passagem pelo pronto socorro). Desta forma, a amostra final foi composta por 179 idosos.

Para a coleta de dados foi elaborado instrumento estruturado com questões fechadas, constituído por variáveis sociodemográficas, clínicas, laboratoriais e desfechos dos idosos hospitalizados com COVID-19. Os dados coletados foram armazenados e organizados em arquivo Microsoft Excel® para a codificação e tabulação e importados para o Statistical Package for the Social Sciences (SPSS ${ }^{\circledR}$ ).

O estudo foi aprovado pelo Comitê de Ética em Pesquisa da Universidade Federal do Paraná, conforme parecer consubstanciado número 4.624.299. Foi dispensado a necessidade de obter-se o termo de consentimento livre esclarecido, em razão do tipo de estudo e delineamento da coleta dados, na qual foram utilizados dados secundários. Assegurou-se a confidencialidade com relação à identidade dos pacientes e fidedignidade das informações oriundas dos relatórios e boletins do Núcleo de Epidemiologia.

\section{Resultados}

Da amostra composta por 179 idosos, a idade deles variou entre 60 a 97 anos (média de 71,99 anos $\pm 8,96$ ) os gêneros tiveram prevalências bem próximas: feminino $(49,7 \%)$ e masculino $(50,3 \%)$ e residentes no estado do Paraná ( $\mathrm{n}=176 ; 98,3 \%)$, apenas n=3 idosos tinham residência distribuída entre os estados de São Paulo e Santa Catarina, respectivamente (n=2; 1,1\%; $\mathrm{n}=1 ; 0,6 \%)$.

Observa-se na Tabela 1 que os principais sinais e sintomas apresentados pelos idosos hospitalizados foram dispneia $(n=112 ; 62,6 \%)$, tosse $(n=87 ; 48,6 \%)$, febre $(n=67 ; 37,4 \%)$, dessaturação de oxigênio menor que $95 \%(n=41 ; 22,9)$, fadiga $(n=39$; $21,8 \%)$, seguidos de mialgia $(\mathrm{n}=27 ; 15,1 \%)$ e mal estar $(\mathrm{n}=24 ; 13,4 \%)$.

Tabela 1 - Distribuição dos sinais, sintomas e morbidades apresentados pelos pacientes idosos hospitalizados com Covid-19. Curitiba, Paraná, 2021.

\begin{tabular}{lcc}
\hline \multirow{2}{*}{\multicolumn{1}{c}{ Sinais e Sintomas }} & \multicolumn{2}{c}{ Sim } \\
\cline { 2 - 3 } & $\mathbf{n}$ & $\mathbf{\%}$ \\
\hline Dispneia & 112 & $62,6 \%$ \\
Tosse & 87 & $48,6 \%$ \\
Febre & 67 & $37,4 \%$ \\
Dessaturação (Saturação de oxigênio < 95\%) & 41 & $22,9 \%$ \\
Fadiga & 39 & $21,8 \%$ \\
Mialgia & 27 & $15,1 \%$ \\
Mal estar & 24 & $13,4 \%$ \\
Cefaleia & 23 & $12,8 \%$ \\
Disgeusia & 22 & $12,3 \%$ \\
Diarreia & 15 & $8,4 \%$ \\
Esforço respiratório & 14 & $7,8 \%$ \\
Odinofagia & 12 & $6,7 \%$ \\
Náusea & 10 & $5,6 \%$ \\
Anosmia & 6 & $3,4 \%$ \\
Calafrio & 5 & $2,8 \%$ \\
Rinorreia & 5 & $2,8 \%$ \\
Vômito & 4 & $2,2 \%$ \\
Padrão intestinal alterado & 3 & $1,7 \%$ \\
Congestão nasal & 3 & $1,7 \%$ \\
\hline
\end{tabular}

Fonte: Autores (2021)

Além disso, em relação as morbidades, $88,8 \%(\mathrm{n}=159)$ dos idosos apresentavam pelo menos um tipo, sendo prevalente em 122 idosos a Hipertensão Arterial Sistêmica (HAS) (68,2\%), seguida pela diabetes Mellitus em 62 idosos (34,6\%) e cardiovascular em 40 (22,3\%), destaca-se que nos registros apresentava-se categoria de doença cardiovascular separadamente 
da HAS. Entre as outras morbidades apresentadas pelos idosos hospitalizados com Covid-19 estão: neurológica ( $\mathrm{n}=14 ; 7,8 \%$ ); obesidade ( $\mathrm{n}=11 ; 6,1 \%)$; pneumonia crônica $(\mathrm{n}=9 ; 5 \%)$; doença renal $(\mathrm{n}=9 ; 5 \%)$; imunodepressão $(\mathrm{n}=6 ; 3,4 \%)$; asma $(\mathrm{n}=1,0,6 \%)$ e doença hematológica $(n=1 ; 0,6 \%)$.

Verifica-se na Tabela 2 que, 121 (67,6\%) idosos apresentaram opacidades na tomografia de tórax, 31 (17,3\%) tomografia mista, opacidades e consolidações, 5 (2,8\%) consolidações e 4 (2,2\%) infiltrado intersticial. Quanto à necessidade de suporte de oxigênio, 142 (79,3\%) fizeram utilização no ambiente hospitalar.

Quanto às necessidades de tratamento, 40 (22,3\%) idosos precisaram de sedação, 31 (17,3\%) foram submetidos a ventilação mecânica, 28 (15,6\%) de forma invasiva e $3(1,7 \%)$ não invasiva. A utilização da posição de prona, foi realizada com $101(56,4)$ idosos, sendo que muitos idosos apresentam dificuldades para tolerar este posicionamento, apresentando principalmente instabilidades hemodinâmicas. A maioria dos idosos foi identificada com transmissão do tipo comunitária da COVID-19, representados por 175 (97,8\%), esses dados podem ser visto na Tabela 2.

Tabela 2 - Distribuição de frequência das características clínicas dos pacientes idosos hospitalizados com Covid-19. Curitiba, Paraná, Brasil. 2021.

\begin{tabular}{lcc}
\hline Características Clínicas e Epidemiológicas & n & \% \\
\hline Tomografia de tórax & 121 & $67,6 \%$ \\
\hline Opacidades & 31 & $17,3 \%$ \\
Misto & 12 & $6,7 \%$ \\
Não Realizado & 6 & $3,4 \%$ \\
Outros & 5 & $2,8 \%$ \\
Consolidações & 4 & $2,2 \%$ \\
Infiltrado intersticial & & \\
\hline Suporte de O2 & 115 & $64,2 \%$ \\
\hline Sim, não invasivo & 27 & $15,1 \%$ \\
Sim, invasivo & 37 & $20,7 \%$ \\
Não & & \\
\hline Ventilação Mecânica & 31 & $17,3 \%$ \\
\hline Sim & 151 & $84,4 \%$ \\
Não & & \\
\hline Tipo de Ventilação & 28 & $15,6 \%$ \\
\hline Invasiva & 3 & $1,7 \%$ \\
Não Invasiva & 148 & $82,7 \%$ \\
Não utilizado & & \\
\hline Prona & 101 & $56,4 \%$ \\
\hline Sim & 78 & $43,6 \%$ \\
Não & & \\
\hline Sedação & 40 & $22,3 \%$ \\
\hline Sim & 139 & $77,7 \%$ \\
\hline Não & & \\
\hline Tipo de transmissão COVID-19 & & \\
\hline Comunitária & & \\
Nosocomial & & \\
\hline & & \\
\hline
\end{tabular}

Fonte: Autores (2021). 
Durante o período em que os pacientes estiveram hospitalizados, houve média de pressão parcial de Oxigênio (PaO2) de 102,79 $\pm 47,47$, e em relação ao exame D-Dímero a média foi de 2016,98 $\pm 1994,94$. Dos idosos que tiveram desfechos de óbito, a média D-Dímero foi de 5022,29.

As escalas aplicadas, verificou-se: APACHE II (Acute Physiology and Chronic Health Evaluation II) $(12,59 \pm 6,13)$, Mortalidade predita APACHE II $(4,59 \pm 10,04)$, Mortalidade corrigida APACHE II $(1,38 \pm 8,46)$, APACHE IV (Acute Physiology and Chronic Health Evaluation IV) $(18,43 \pm 23,10)$, Mortalidade predita APACHE IV $(3,07 \pm 7,88)$, e Tempo de internação predita APACHE IV $(1,13 \pm 2,07)$.

Ademais, em relação ao suporte clínico, constata-se, que houve predomínio do uso de anticoagulante (n=167; 93,3\%) e corticoide ( $\mathrm{n}=159 ; 88,8 \%)$, seguido de outras opções de suporte medicamentoso, entre elas: droga vasoativa ( $\mathrm{n}=46 ; 25,7 \%)$; tamiflu (n=34;19,0\%); ivermectina $(n=1 ; 0,6 \%)$ e antifúngico $(n=1 ; 0,6 \%)$.

Enquanto no desfecho clínico, além dos idosos que foram transferidos para outro hospital 11 (6,1\%), verificou-se que 127 (71\%) receberam alta hospitalar e 41 (22,9\%) foram a óbito durante a internação, 100\% deles na primeira hospitalização.

Dos idosos que apresentaram desfecho clínico de alta hospitalar (70,9\%) destacaram-se os seguintes sinais e sintomas: dispneia (41,9\%), tosse (34,6\%), seguido por febre (26,3\%). Em idosos com o desfecho óbito verificou-se: dispneia (15,6\%), tosse $(11,7 \%)$ seguido por febre $(9,5 \%)$, (Tabela 3$)$.

Tabela 3 - Comparação entre os sinais e sintomas dos idosos hospitalizados com COVID-19 e os desfechos clínicos. Curitiba, Paraná, Brasil. 2021

\begin{tabular}{|c|c|c|c|c|}
\hline \multirow{3}{*}{$\begin{array}{l}\text { Presença de sinais e sintomas e os } \\
\text { conforme desfechos clínicos }\end{array}$} & \multirow{2}{*}{\multicolumn{2}{|c|}{$\begin{array}{c}\text { Altas } \\
\text { Sim }\end{array}$}} & \multirow{2}{*}{\multicolumn{2}{|c|}{$\begin{array}{c}\text { Óbitos } \\
\text { Sim }\end{array}$}} \\
\hline & & & & \\
\hline & $\mathbf{n}$ & $\%$ & $\mathbf{n}$ & $\%$ \\
\hline Dispneia & 75 & $41,9 \%$ & 28 & $15,6 \%$ \\
\hline Tosse & 62 & $34,6 \%$ & 21 & $11,7 \%$ \\
\hline Febre & 47 & $26,3 \%$ & 17 & $9,5 \%$ \\
\hline Dessaturação (Saturação de < 95\%) & 23 & $12,8 \%$ & 15 & $8,4 \%$ \\
\hline Fadiga & 22 & $12,3 \%$ & 14 & $7,8 \%$ \\
\hline Mialgia & 18 & $10,1 \%$ & 7 & $3,9 \%$ \\
\hline Mal estar & 21 & $11,7 \%$ & 1 & $0,6 \%$ \\
\hline Cefaleia & 12 & $6,7 \%$ & 10 & $5,6 \%$ \\
\hline Disgeusia & 14 & $7,8 \%$ & 7 & $3,9 \%$ \\
\hline Diarreia & 9 & $5,0 \%$ & 6 & $3,4 \%$ \\
\hline Esforço respiratório & 8 & $4,5 \%$ & 6 & $3,4 \%$ \\
\hline Odinofagia & 7 & $3,9 \%$ & 2 & $1,1 \%$ \\
\hline Náusea & 6 & $3,4 \%$ & 3 & $1,7 \%$ \\
\hline Anosmia & 3 & $1,7 \%$ & 2 & $1,1 \%$ \\
\hline Calafrio & 2 & $1,1 \%$ & 3 & $1,7 \%$ \\
\hline Rinorreia & 4 & $2,2 \%$ & 1 & $0,6 \%$ \\
\hline Vômito & 4 & $2,2 \%$ & 1 & $0,6 \%$ \\
\hline Padrão intestinal alterado & 0 & $0,0 \%$ & 3 & $1,7 \%$ \\
\hline Congestão nasal & 0 & $0,0 \%$ & 3 & $1,7 \%$ \\
\hline
\end{tabular}

*Nesta descrição não foram incluídos os pacientes com desfecho transferência. Fonte: Autores (2021). 
Quanto à prevalência de morbidades, nos idosos que obtiveram desfecho de alta, evidenciaram-se três principais patologias: hipertensão arterial sistêmica (43,6\%), diabetes Mellitus (24,0\%), seguida por doença cardiovascular (14,0\%). No desfecho óbito prevaleceram as mesmas três principais: HAS (75,6\%), diabetes Mellitus $(34,1 \%)$ seguido por doença cardiovascular (22,0\%), (Tabela 4).

Tabela 4 - Comparação entre as doenças dos idosos hospitalizados com COVID-19 e os desfechos clínicos. Curitiba, Paraná, Brasil. 2021

\begin{tabular}{|c|c|c|c|c|c|c|c|c|}
\hline \multirow{3}{*}{ Morbidades e os desfechos clínicos } & \multicolumn{4}{|c|}{ Altas $(n=127)$} & \multicolumn{4}{|c|}{ Óbitos $(\mathrm{n}=41)$} \\
\hline & \multicolumn{2}{|c|}{$\mathrm{Sim}$} & \multicolumn{2}{|c|}{ Não } & \multicolumn{2}{|c|}{ Sim } & \multicolumn{2}{|c|}{ Não } \\
\hline & $\mathbf{n}$ & $\%$ & $\mathbf{N}$ & $\%$ & $\mathbf{n}$ & $\%$ & $\mathbf{n}$ & $\%$ \\
\hline HAS & 78 & $43,6 \%$ & 49 & $27,4 \%$ & 31 & $75,6 \%$ & 10 & $24,4 \%$ \\
\hline Diabetes Mellitus & 43 & $24,0 \%$ & 84 & $46,9 \%$ & 14 & $34,1 \%$ & 27 & $65,9 \%$ \\
\hline Cardiovascular & 25 & $14,0 \%$ & 102 & $57,0 \%$ & 9 & $22,0 \%$ & 32 & $78,0 \%$ \\
\hline Neurológica & 6 & $3,4 \%$ & 121 & $67,6 \%$ & 7 & $17,1 \%$ & 34 & $82,9 \%$ \\
\hline Doença renal & 2 & $1,1 \%$ & 125 & $69,8 \%$ & 5 & $12,2 \%$ & 36 & $87,8 \%$ \\
\hline Obesidade & 7 & $3,9 \%$ & 120 & $67,0 \%$ & 3 & $7,3 \%$ & 38 & $92,7 \%$ \\
\hline Imunodepressão & 4 & $2,2 \%$ & 123 & $68,7 \%$ & 3 & $7,3 \%$ & 38 & $92,7 \%$ \\
\hline Pneumopatia crônica & 7 & $3,9 \%$ & 120 & $67,0 \%$ & 2 & $4,9 \%$ & 39 & $95,1 \%$ \\
\hline Asma & 1 & $0,6 \%$ & 126 & $70,4 \%$ & 0 & $0,0 \%$ & 41 & $100,0 \%$ \\
\hline Doença hematológica & 1 & $0,6 \%$ & 126 & $70,4 \%$ & 0 & $0,0 \%$ & 41 & $100,0 \%$ \\
\hline
\end{tabular}

*Nesta descrição não foram incluídos os pacientes com desfecho transferência. Fonte: Autores (2021).

Na Tabela 5 verifica-se que a média de idade no desfecho alta foi de 70,4 anos, com predomínio do sexo masculino (53,54\%), já nos óbitos a média de idade atingiu 76,6 anos e a maioria do sexo feminino (58,54\%). A alteração no exame de tomografia com aspecto em opacidades, para o desfecho alta foi de $66,79 \%$ e nos óbitos de $73,2 \%$. A maioria das altas fizeram uso de suporte de oxigênio $(72,44 \%)$ e destes 5,5\% fizeram uso de ventilação mecânica; nos óbitos observa-se a necessidade de oxigenoterapia em $97,53 \%$ dos idosos e foram entubados $58,54 \%$. Em relação à posição de prona, no desfecho alta foram pronados 101 idosos $(79,53 \%)$ e dos que evoluíram a óbito 25 idosos (60,98\%) (Tabela 5). 
Tabela 5 - Comparação entre as principais características dos idosos hospitalizados com COVID-19 conforme e os desfechos clínicos. Curitiba, Paraná, Brasil. 2021

\begin{tabular}{|c|c|c|c|c|}
\hline \multirow{2}{*}{$\begin{array}{c}\text { Principais Características em Relação aos } \\
\text { Desfechos }\end{array}$} & \multicolumn{2}{|c|}{ Altas $(n=127)$} & \multicolumn{2}{|c|}{ Óbitos (n=41) } \\
\hline & Média & Desvio Padrão & Média & Desvio Padrão \\
\hline Idade & 70,4 & 7,8 & 76,6 & 9,74 \\
\hline$\overline{\mathrm{PaO} 2}$ & 103 & 48 & 107 & 46 \\
\hline D-Dímero & 1727,7 & 2166,2 & 5022,1 & 2309,03 \\
\hline$\overline{\text { Sexo }}$ & $\mathbf{N}$ & $\%$ & $\mathbf{n}$ & $\%$ \\
\hline Feminino & 59 & $46,46 \%$ & 24 & $58,54 \%$ \\
\hline Masculino & 68 & $53,54 \%$ & 17 & $41,46 \%$ \\
\hline \multicolumn{5}{|l|}{ Tomografia de tórax } \\
\hline Opacidades & 85 & $66,9 \%$ & 30 & $73,2 \%$ \\
\hline \multicolumn{5}{|l|}{ Suporte de O2 } \\
\hline Sim & 92 & $72,44 \%$ & 40 & $97,56 \%$ \\
\hline Não & 35 & $27,56 \%$ & 1 & $2,44 \%$ \\
\hline \multicolumn{5}{|l|}{ Tipo de Suporte de O2 } \\
\hline Sim, não invasivo & 85 & $66,93 \%$ & 24 & $58,54 \%$ \\
\hline Sim, invasivo & 7 & $5,51 \%$ & 16 & $39,02 \%$ \\
\hline Não & 35 & $27,56 \%$ & 1 & $2,44 \%$ \\
\hline \multicolumn{5}{|l|}{ Ventilação Mecânica } \\
\hline Sim & 7 & $5,51 \%$ & 24 & $58,54 \%$ \\
\hline Não & 120 & $94,49 \%$ & 17 & $41,46 \%$ \\
\hline \multicolumn{5}{|l|}{ Prona } \\
\hline Sim & 101 & $79,53 \%$ & 25 & $60,98 \%$ \\
\hline Não & 26 & $20,47 \%$ & 16 & $39,02 \%$ \\
\hline
\end{tabular}

*Nesta descrição não foram incluídos os pacientes com desfecho transferência. Fonte: Autores (2021).

\section{Discussão}

No presente estudo a idade média observada entre os idosos hospitalizados com COVID-19 foi de 71,9 anos, média semelhante foi apontada entre pacientes hospitalizados em Wuhan, na China (Backer et al., 2020; Chen, Ling et al., 2020; Chen, Du et al., 2020; Zhou et al., 2020) e inferior a obtida em pacientes hospitalizados em Nova Iorque, nos Estados Unidos (média de idade 75 anos) e nos admitidos na Lomabardia, Itália, (média de idade 78 anos). As diferenças podem ser explicadas pelos perfis etários das populações gerais desses países, pois a população do Brasil e da China possuem um menor percentual de pessoas idosas em comparação aos Estados Unidos e Itália (Onu, 2019).

O predomínio do sexo masculino (50,3\%), embora bem prevalência próxima do sexo feminino, também foi identificado nos estudos op cit, realizados na China e nos Estados Unidos, com percentual ainda mais elevado entre os pacientes admitidos na Lombardia (82\%). Visto que o sexo masculino corresponde aproximadamente metade das populações desses países China (Backer et al., 2020; Chen, Ling et al., 2020; Chen, Du et al., 2020; Zhou et al., 2020; Onu, 2019).

Quanto aos sinais e sintomas, estudos realizados na China evidenciaram distribuição de dispneia (29\%), tosse (79\%), febre $(94 \%)$, fadiga $(44 \%)$, dessaturação de oxigênio (saturação < 95\%), corroborando às taxas encontradas nesta pesquisa, na qual $62,6 \%$ dos idosos apresentavam dispneia, seguido de tosse em 48,6\%, febre em 37,4\%, 22,9 com saturação periférica de oxigênio $\leq 95 \%$ e fadiga em 21,8,\% (Zhou et al., 2020; Zhang et al., 2020; Yang et al., 2020).

Os idosos que apresentaram dessaturação e hipoxemia mostraram dificuldades para se manterem na posição prona. Destaca-se que para o idoso com COVID-19, a indicação da posição prona requer avaliação prévia de aspectos gerontogeriátricos, pois há necessidade de se considerar as alterações fisiológicas que ocorrem no processo de envelhecimento 
(Bitencourt, 2021). A saturação periférica de oxigênio $\leq 95 \%$ são importantes fatores associados ao óbito segundo a literatura atual (Zhang et al., 2020).

Em relação às morbidades, no presente estudo $88,8 \%$ dos idosos apresentavam pelo menos um tipo de doença, sendo prevalente em 122 idosos a hipertensão arterial sistêmica (HAS), 68,2\% dos idosos apresentavam. Achado condizente com vários autores, que indicam variação inclusive quanto à inclusão ou não da hipertensão arterial entre as doenças cardiovasculares (essa inclusão não ocorreu neste estudo) de maior incidência, seguida de diabetes Mellitus em 62 idosos (34,6\%), próxima à encontrada em estudos norte-americanos (33,8 a 38,4\%), maior do que no Reino Unido $(28,1 \%)$ e bem superior à estimativa para a população geral de adultos brasileiros (6,2\%) (Guan, Tang et al., 2020; Richardson et al., 2020; Docherty et al., 2020; Suleyman et al., 2020; Niquini et al., 2020; Gil-Rodrigo et al., 2020; Zheng et al., 2020; Gupta et al., 2020; Grasseli et al., 2020).

Em idosos que obtiveram desfecho de alta e óbito também foram prevalentes a HAS, seguida pela diabetes Mellitus. Quanto à presença de morbidades, observou-se grande variação na literatura consultada, desde 65\% dos casos em estudo chinês (Bourouiba et al., 2020; Guan, Li et al., 2020; Guan, Ni et al., 2020) a 80,5\% dos casos internados em UTIs na Itália (Gupta et al., 2020) e 87\% dos casos hospitalizados no Reino Unido (Docherty et al., 2020); e até cerca de $96 \%$ dos casos hospitalizados em Nova Iorque e Detroit, Estados Unidos (Richardson et al., 2020; Suleyman et al., 2020). Quanto à prevalência de diabetes mellitus e doenças cardiovasculares, entre os pacientes hospitalizados por SRAG-COVID no Brasil, foi superior às estimativas para a população geral brasileira, ressaltando a hipótese de que esse grupo apresenta maior chance de ser hospitalizado pela doença (Niquini et al., 2020).

As morbidades mais citadas na literatura vigente foram as cardiovasculares, hipertensão arterial, diabetes mellitus, obesidade, doenças respiratórias, doença renal crônica e câncer (Zhou et al., 2020; Docherty et al., 2020; Suleyman et al., 2020; Niquini et al., 2020; Gil-Rodrigo et al., 2020; Gupta et al., 2020; Zheng et al., 2020; Grasseli et al., 2020). Todas relacionadas a maior risco de complicações da COVID-19, podendo coexistir em mesmo indivíduo. A existência de mais de uma comorbidade está associada a elevação do risco para pior prognóstico (Ministério da Saúde, 2020d; Guan, Tang et al., 2020).

No Brasil, os dados apontam que 69,3\% dos óbitos por COVID-19 ocorreram em pessoas com mais de 60 anos e destes, 64\% apresentavam ao menos uma morbidade (Ministério da Saúde, 2020c). Situação semelhante é reportada por Livingston e Bucher (2020) em estudo realizado na China, com pacientes infectados pelo coronavírus da Síndrome Respiratória Aguda Grave (SARS-CoV), inclusive que necessitaram de admissão em UTI (Livingston \& Bucher, 2020).

Em estudo de revisão sistemática e meta-análise apontou que entre os pacientes com COVID-19, 20,3\% necessitaram de cuidados de unidade de terapia intensiva (Rodriguez-Morales et al., 2020). Neste estudo, 94 pacientes necessitaram de cuidados na unidade de terapia intensiva, representando 52,5\%, e destes 39 (41,4\%) tiveram como desfecho óbito. A média de permanência na UTI foi de 8,8 (mínimo de 1 dia, e máximo de 40 dias), a média de permanência na enfermaria foi de 4,94 dias (mínimo de 1 dia e máximo de 76 dias).

A duração mediana de ventilação mecânica (VM) no presente estudo foi de 14 dias, em estudo realizado na China por Wang et al (2020) foi de 12 dias, sendo referenciado que estudos prévios reforçam essa duração na COVID-19 grave. Quanto aos achados de tomografia, houve predomínio do padrão de opacidades (67,6\%). Conforme Huang, Wang et al. (2020), corroborando aos achados observou-se, em um dos primeiros estudos de Wuhan (90 pacientes), o percentual de opacidade em $72 \%$ dos idosos (Xu et al., 2020). Em outro estudo de coorte, que também foi desenvolvido na China, as opacidades em vidro fosco foram observadas em $77 \%$ dos pacientes (Song et al., 2020).

Os idosos que necessitaram de UTI, 25,7\% fizeram uso de droga vasoativa, os guidelines indicam abordagem conservadora na oferta de fluidos em pacientes que necessitam de hospitalização em UTI, sendo que as metas consistem em alvo de PAM acima de 60 (principalmente pacientes $\geq 65$ anos) ou $65 \mathrm{mmHg}$, com uso precoce de vasopressores (Nacional Institutes of Health, 2020). 
Para o tratamento medicamentoso, inicialmente na pandemia, houve receio no uso de anticoagulante e corticosteróides, já que, foram considerados os dados de estudos realizados nas epidemias de H1N1 e MERS. No entanto, após a publicação dos estudos RECOVERY, CODEX, e da metanálise da OMS que incluiu outros cinco estudos randomizados, o uso da dexametasona, se tornou recomendação formal de utilização para COVID-19 (Recovery, 2020; Who, 2020).

Quanto à medicação corticóide, 159 idosos fizeram uso $(88,8 \%)$ e, 16 (93,3\%) utilizaram anticoagulação profilática. Destaca-se que, indivíduos mais graves costumam apresentar outros fatores de risco trombótico, como imobilização prolongada, acesso venoso profundo e uso de determinados medicamentos e hemocomponentes (Wang et al., 2020). A COVID-19 desencadeia estado de hipercoagulabilidade secundário à COVID-19, desta forma é importante a avaliação da elegibilidade desta profilaxia e quando indicada deve ser prescrita (Wijaya et al., 2020).

Quando existem alterações no processo de coagulação é possível que exista maior quantidade de dímero-D circulante. Para os idosos com COVID-19 a presença de D-dímero elevado na admissão está associada ao fator de pior prognóstico. O estudo realizado no hospital Fuyang (China) observou esse marcador elevado em $81 \%$ dos pacientes que faleceram com COVID19, porém, apesar de se correlacionar a maior gravidade, a presença de D-dímero elevado não indica pesquisa de evento tromboembólico, a menos que exista suspeita clínica desta condição (Gao et al., 2021). Esses dados convergem ao presente estudo, dado que, entre os 179 idosos a média D-dímero foi de 2016,98, porém este valor para os idosos com desfechos de óbito observou-se média de 5022,29.

Referente à prescrição de antimicrobianos foi realizada para 156 idosos (87,2\%), destes, 22 desenvolveram infecção nosocomial secundária, representando taxa de 12,29\%, nos demais o antimicrobiano foi iniciado já na admissão hospitalar. É importante o julgamento clínico individualizado, associado a marcadores de infecções que podem ajudar a decidir o melhor tratamento para cada idoso, evitar a seleção de cepas resistentes por uso indiscriminado de antimicrobianos, visto que cepas multirresistentes aumentam a letalidade (Livingston \& Bucher, 2020).

Neste estudo a letalidade foi de 22,9\% na primeira hospitalização. Sendo que, as taxas gerais de letalidade no estado do Paraná, foram de aproximadamente $2 \%$ a $3 \%$, porém atingiram $21 \%$ entre pacientes hospitalizados, e em idosos a letalidade foi de 38,8\% de acordo com o Boletim da SESA de dezembro de 2020 (Secretaria Estadual de Saúde do Paraná, 2020). A análise da letalidade por grupo etário apresentada pela Itália apontou aumento principalmente dos óbitos após os 70 anos, sendo de 12,5\% na faixa de 70-79 anos, 19,7\% na faixa de 80-89 anos e 22,7\% após 90 anos (Livingston \& Bucher, 2020). Em Curitiba a taxa de letalidade da COVID-19 em idosos em 2020 foi de 12,57 (Secretaria Municipal de Saúde de Curitiba, 2020).

Quanto ao APACHE II, avaliação e classificação do índice de gravidade da doença, e tem como objetivo principal a descrição quantitativa do grau de disfunção orgânica de pacientes gravemente enfermos, gravidade que é traduzida em valor numérico a partir das alterações clínicas e laboratoriais existentes ou do tipo/número de procedimentos utilizados (Baltussen \& Kindler, 2004). No presente estudo verificou-se que o índice APACHE II obteve a média de 12,59 ( \pm DP: 6,13). Esse dado é significativo para prever o risco de mortalidade em pacientes com COVID-19, pois no momento da admissão fornece a primeira evidência direta. Por vezes, considerado um escore mais baixo, mas no decorrer da hospitalização na UTI a pessoa idosa pode evoluir para um pior prognóstico na forma da COVID-19 grave, sendo estático este escore e direcionado apenas para a admissão.

Chen, Li et al (2020) destaca que, nenhuma ferramenta de prognóstico está disponível para a previsão de gravidade e mortalidade pela COVID-19. Em estudo retrospectivo o autor comparou a consistência de três escalas para predizer gravidade e mortalidade: o PSI (Índice de Gravidade da Pneumonia), o CURB (confusão, ureia, frequência respiratória, pressão arterial, idade $\geqslant 65$ ) e o APACHE II, sugerindo o PSI e CURB-65 úteis para prever gravidade e mortalidade, o APACHE II também pode ser utilizado, porém com menor especificidade, quando comparado ao PSI e CURB-65 (Chen, Li et al., 2020). 


\section{Conclusão}

Dos dados de 179 idosos extraídos da fonte Núcleo de Epidemiologia Hospitalar conclui-se que a prevalência de morbidades, nos idosos que obtiveram desfecho de alta, evidenciaram-se três principais patologias: hipertensão arterial sistêmica (43,6\%), diabetes Mellitus (24,0\%), seguida por doença cardiovascular (14,0\%). No desfecho óbito prevaleceram as mesmas três principais: HAS (75,6\%), diabetes Mellitus $(34,1 \%)$ seguido por doença cardiovascular $(22,0 \%)$.

Verificou-se que a média de idade no desfecho alta foi de 70,4 anos, com predomínio do sexo masculino (53,54\%), já nos óbitos a média de idade atingiu 76,6 anos e a maioria do sexo feminino (58,54\%). A alteração no exame de tomografia com aspecto em opacidades, para o desfecho alta foi de 66,79\% e nos óbitos de 73,2\%. A maioria das altas fizeram uso de suporte de oxigênio $(72,44 \%)$ e destes 5,5\% fizeram uso de ventilação mecânica; nos óbitos observa-se a necessidade de oxigenoterapia em 97,53\% dos idosos e foram entubados 58,54\%. Em relação à posição de prona, no desfecho alta foram pronados 101 idosos $(79,53 \%)$ e dos que evoluíram a óbito 25 idosos $(60,98 \%)$.

Destaca-se a necessidade de estudos futuros que apontem as relações entre as variáveis de interesse do presente estudo (morbidades, sintomas relacionando com a alta e óbito). Sugere-se os estudos observacionais do tipo coorte, os quais viabilizam níveis de evidência sobre a relação entre as variáveis em estudo, e, consequentemente oportunizam inferências mais precisas, para aprofundar os dados estatísticos relacionados aos idosos hospitalizados com COVID-19, correlacionando com informações sobre os desfechos clínicos, destacando fatores relacionados relevantes para a prática clínica.

\section{Referências}

Aquino E. M. L., Silveira I. H., Pescarini J., Aquino R., \& Souza-Filho J. Á. (2020). Medidas de distanciamento social no controle da pandemia de COVID-19: potenciais impactos e desafios no Brasil. Ciência \& Saúde Coletiva, 25(Supl.1), 2423-2446. https://doi.org/10.1590/1413-81232020256.1.10502020

Backer J., Klinkenberg D. \& Wallinga J. (2020). Incubation period of 2019 novel coronavirus (2019-nCoV) infections among travellers from Wuhan, China, 20-28. EuroSurveillance, 25(5):2000062. https://doi.org/10.2807/1560-7917.ES.2020.25.5.2000062

Baltussem A. \& Kindler C. H. (2004). Citation classics in critical care medicine. Intensive Care Med, 30(5):90210. https://pubmed.ncbi.nlm.nih.gov/14985952/ doi: $10.1007 / \mathrm{s} 00134-004-2195-7$

Barbosa I. R., Galvão M. H. R., Sousa T. A., Gomes M. S., Medeiros A. A., \& Lima K. (2020). Incidência e mortalidade por COVID-19 na população idosa brasileira e sua relação com indicadores contextuais: um estudo ecológico. Bras. Geriatr. Gerontol, 23(1):e200171. https://pesquisa.bvsalud.org/portal/resource/pt/biblio-1117772

Bitencourt, G. R., Almeida A. R., Corrêa B. L. M. A. L., Rocha G. S., Souza R. S., Couto V. M., \& Santana R. F. (2021). Pronação do Idoso na Covid-19: Considerações de Enfermagem Gerontológica. In: Santana RF (Org.). Enfermagem gerontológica no cuidado do idoso em tempos da COVID 19. Brasília, DF: Editora Aben, 171 p. (Serie Enfermagem e Pandemias, 5). https://doi.org/10.51234/aben.21.e05.c15

Bourouiba L (2020). Turbulent gas clouds and respiratory pathogen emissions - Potential Implications for Reducing Transmission of Covid 19. Jama, 21;323(18):1837-1838. 10.1001/jama.2020.4756

Cellard A. (2012). Análise documental. In Poupart J., Deslauriers J. P., Groulx L. H., Laperrière A., Mayer R., Pires À. A pesquisa qualitativa: enfoques epistemológicos e metodológicos. (3a ed., pp. 295-316), Rio de Janeiro: Vozes.

Chen J., Qi T., Liu L., Ling Y., Qian Z., Li T., Li F., Xu Q., Zhang Y., Xu S., Song Z., Zeng Y., Shen Y., Shi Y., Zhu T., \& Lu H. (2020). Clinical progression of patients with COVID-19 in Shanghai, China. J Infect, 80(5):e1-e6. https://doi: 0.1016/j.jinf.2020.03.004.

Chen J., Qi T., Liu L., Du H., Lin H., Chen C., Rao S., Yu R., Wang J., Xue Z., Zhang Y., \& Xie Y. (2020). Clinical progression of patients with COVID-19 in Shanghai, China. European Journal Inflammation, 80(5):e1-e6. https://journals.sagepub.com/doi/pdf/10.1177/20587392211027083

Docherty A. B., Harrison E. M., Green C. A., Hardwick H. E., Pius R., Norman L., Holden K. A., Read J. M., Dondelinger F., Carson G., Merson L., Lee J., Plotkin D., Sigfrid L., Halpin S., Jackson C., Gamble C., Horby P. W., Nguyen-Van-Tam J. S., \& K., Semple M. G. (2020). Features of 20 133 hospitalized UK patients with COVID-19 using the ISARIC WHO Clinical Characterization Protocol: prospective observational cohort study. BMJ, 369:m1985. 10.1136/bmj.m1985

Gao Y., Li T., Han M., Li X., Xu Y., Zhu Y., Liu Y., Wang X., \& Wang L. (2020). Diagnostic utility of clinical laboratory data determinations for patients with the severe Covid-19. J Med Virol, 92(7):791-796. 10.1002/jmv.25770

GIL, A. C. (2008). Métodos e técnicas de pesquisa social. Atlas.

Gil-Rodrigo A., Miró Ò., Piñera P., Jiménez S., Martín A., Martín-Sánchez F. J., Jacob J., Guardiola J. M., García-Lamberechts E. J., Espinosa B., Mojarro E. M., Tejera M. G., Serrano L., Agüera C., Soy E., Llauger L., Juan M. A., Palau A., \& Alquézar-Arbé A. (2020). Analysis of clinical characteristics and outcomes 
in patients with COVID-19 based on a series of 1000 patients treated in Spanish emergency departments. Emergencias (Sant Vicenç dels Horts), 32(4):233-41. https://pubmed.ncbi.nlm.nih.gov/32692000/

Grasselli G., Greco M., Zanella A., Albano G., Antonelli M., Bellani G., Bonanomi E., Cabrini L., Carlesso E., Castelli G., Cattaneo S., Cereda D., Colombo S., Coluccello A., Crescini G., Molinari A. F., Foti G., Fumagalli R., Iotti G. A., \& Cecconi M. (2020). Risk factors associated with mortality among patients with COVID-19 in intensive care Units in Lombardy, Italy. JAMA Intern Med, 3];180(10):1345- 55. 10.1001/jamainternmed.2020.3539

Guan W. J, Liang W. H., Zhao Y., Liang H. R., Chen Z. S., Li Y. M., Liu X. Q., Chen R. C., Tang C. L., Wang T., Ou C. Q., Li L., Chen P. Y., Sang L., Wang W., Li J. F., Li C. C., Ou L. M., Cheng B., \& He J. X. (2020). Comorbidity and its impact on 1590 patients with COVID-19 in China: a nationwide analysis. Eur Respir J., 55(5):2000547. https://doi. org/10.1183/13993003.00547-2020

Guan W. J., Liang W. H., Zhao Y., Liang H. R., Chen R. C., Tang C. L., Wang T., Ou C. Q., Li L., Chen P. Y., Sang L., Wang W., Li J. F., Li C. C., Ou L. M., Cheng B., Xiong W., Ni Z. Y., Xiang J., \& He J. X. (2020). China Medical Treatment Expert Group for COVID-19. Comorbidity and its impact on 1590 patients with COVID-19 in China: a nationwide analysis. Eur Respir J, 55(5):2000547. 10.1183/13993003.00547-2020

Guan W. J., Ni Z. Y., Hu Y., Liang W. H.. Ou C. Q., He J. X., Liu L., Shan H., Lei C. L., Hui D. S. C., Du B., Li L. J., Zeng G., Yuen K. Y., Chen R. C., Tang C. L., Wang T., Chen P. Y., Xiang J., \& Zhong N. S. (2020). Clinical Characteristics of Coronavirus Disease 2019 in China. N Engl J Med, 382:1708-20. 10.1056/NEJMoa2002032

Gupta S., Hayek S. S., Wang W., Chan L., Mathews K. S., Melamed M. L., Brenner S. K., Leonbérg-Yoo A., Schenck E. J., Radbel J., Reiser J., Bansal A., Srivastava A., Zhou Y., Sutherland A., Green A., Shehata A. M., Goyal N., Vijayan A., \& Leaf D. E. (2020). Factors associated with death in critically Ill patientsoronavírusavirus disease 2019 in the US. JAMA Intern Med, 180(11):1436-46. 10.1001/jamainternmed.2020.3596

Hammerschmidt K. S. A. \& Santana R. F. (2020). Saúde do idoso em tempos de pandemia covid-19. Cogitare enfermagem, 25:e72849. https://revistas.ufpr.br/cogitare/article/view/72849/pdf

Huang C., Wang Y., Li X., Ren L., Zhao J., Hu Y., Zhang L., Fan G., Xu J., Gu X., Cheng Z., Yu T., Xia J., Wei Y., Wu W., Xie X., Yin W., Li H., Liu M., \& Cao B. (2020). Clinical features of patients infected with 2019 novel coronavirus in Wuhan, China. Lan - cet, 395:497-506. 10.1016/S0140-6736(20)30183-5

Lakatos, E. M. \& Marconi, M. A. (1191). Técnicas de pesquisa. In Lakato E. M., Marconi, M. A. Fundamentos de Metodologia Científica. (3a ed.), 95-200, Atlas.

Livingston E. \& Bucher K. (2020). Coronavirus Disease 2019 (COVID-19) in Italy. JAMA, 323(14):1335. https://pubmed.ncbi.nlm.nih.gov/32181795/

Ministério da Saúde (BR). (2020a). COVID-19 - Painel Coronavírus. MS. https:// covid.saude.gov.br/

Ministério da Saúde (BR). (2010). Portaria n 2.254, de 5 de agosto de 2010. Institui a Vigilância Epidemiológica em Âmbito Hospitalar, define as competências para a União, os Estados, o Distrito Federal, os Municípios, os critérios para a qualificação das unidades hospitalares de referência nacional e define também o escopo das atividades a serem desenvolvidas pelos Núcleos Hospitalares de Epidemiologia. Diário Oficial da União. Brasília, DF, 6 ago. 2021. Seção 1. p. 55.

Ministério da Saúde (BR). (2020b). Protocolo de Manejo Clínico do Coronavírus (COVID-19) na Atenção Primária à Saúde. (v9). Secretaria de Atenção Primária à Saúde (SAPS). Brasília. https://portaldeboaspraticas.iff.fiocruz.br/biblioteca/protocolo-de-manejo-clinico-docoronavirus-covid-19-na-atencao-primaria-asaude/

Ministério da Saúde (BR). (2020d). Secretaria de Ciência, Tecnologia, Inovação e Insumos Estratégicos em Saúde. Coronavírus COVID-19: diretrizes para diagnóstico e tratamento da COVID-19 [Internet]. Versão 4. Brasília: MS. https://portalarquivos. saude.gov.br/images/pdf/2020/May/08/DiretrizCovid19-v407-05.20h05m.pdf

Ministério da Saúde (BR). (2020c). Secretaria de Vigilância em Saúde. Boletim Epidemiológico Especial 16 . https://www.saude.gov.br/images/pdf/2020/May/21/2020-05-19---BEE16---Boletimdo-COE-13h.pdf

Ministério da Saúde (BR). (2021a). Secretaria de Vigilância em Saúde. Boletim Epidemiológico Especial. Doença pelo Coronavírus COVID-19. Semana Epidemiológica (21 a 27/2/2021). https://www.gov.br/saude/pt br/media/pdf/2021/marco/05/boletim_epidemiologico_covid_52_final2.pdf

Moreira S. V. (2009). Análise documental como método e como técnica. In Duarte J., Barros A., (Orgs). Métodos e técnicas de pesquisa em comunicação. pp. 269-79, Atlas.

National Institutes of Health. (2020). Covid-19 Treatment Guidelines Panel. Coronavirus Disease 2019 (Covid-19). Treatment Guidelines. http://www.covid19treatmentguidelines.nih.gov.

Ng S. C. \& Tilg H. (2020). Covid-19 and the gastrointestinal tract: more than meets the eye. Gut, 69(9):973-974 10.1136/gutjnl-2020-321195

Niquini P. R., Lana R. M., Pacheco A. G., Cruz O. G., Coelho F. C., Carvalho L. M., Villela D. A. M., Gomes M. F. C., \& Bastos L. M. (2020). SRAG por COVID-19 no Brasil: descrição e comparação de características demográficas e comorbidades com SRAG por influenza e com a população geral. Cadernos de Saúde Pública., 36(7):e00149420. https://doi.org/10.1590/0102-311X00149420

Oliveira A. C., Lucas T. C., \& Iquiapaza R. A. (2020). O que a pandemia da covid-19 tem nos ensinado sobre adoção de medidas de precaução? Texto contexto Enferm, 29:e20200106. https://doi.org/10.1590/1980-265x-tce-2020-0106.

Organização das Nações Unidas (ONU) (1950-2020). (2019) World population prospects 2019. Estimates. https://population.un.org/wpp/ Download/Standard/Population 
Recovery Collaborative Group, Horby P., Lim W. S., Emberson J. R., Mafham M., Bell J. L., Linsell L., Staplim N., Brightling C., Ustianowski A., Elmahi E, Prudon B, Green C, Felton T, Chadwick D, Rege K, Fegan C, Chappell LC, Faust SN, Jaki T, \& Landray M (2020). Dexamethasone in Hospitalized Patients withCovid-19. N Engl J Med, 384:693-704. 10.1056/NEJMoa2021436

Richardson S., Hirsch J. S., Narasimhan M., Crawford J. M., Mcginn T., \& Davidson K. W. (2020). COVID-19 Research Consortium. Presenting characteristics comorbidities and outcomes among 5700 patients hospitalized with COVID-19 in the New York City Area. JAMA, 323(20):2052. http://dx.doi.org/10.1001/jama.2020.6775

Rodriguez-Morales J. Á., Cardona-Ospina J. Á., Gutiérrez-Ocampo E., Villamizar- Peña R., Holguin-Riviera Y., Escalera-Antezana J. P., Alvarado-Arnez L. E., Bonilla-Aldana D. K., Franco-Paredes C., Henao-Martinez A. F., Paniz-Mondolfi A., Lagos-Grisales G. J., Ramírez-Vallejo E., Suárez J. Á., Zambrano L. I., Villami-Gómez, W. E., Balbin-Ramon G. J., Rabaan A. A., Harapan H., Dhama K., . \& Sah R. (2020). Clinical, laboratory and imaging features of COVID19: A systematic review and meta-analysis. Travel Medicine and Infectious Disease, 34, 1016232. https://doi.org/10.1016/j.tmaid.2020.101623.

Secretaria Estadual de Saúde do Paraná (SESA). Boletim Epidemiológico. Paraná, Brasil. https://www.saude.pr.gov.br/Pagina/Coronavirus-COVID-19

Secretaria Municipal de Saúde de Curitiba (SMS) (2020). Centro de epidemiologia. Painel COVID 19 Curitiba. Paraná, Brasil. https://datastudio.google.com/u/1/reporting/3f45a263-ae46-45be-9efc-a9e47b358dd4/page/6x9zB

Song F., Shi N., Shan F., Zhang Z., Shen J., Lu H., Ling Y., Jiang Y., \& Shi Y. (2020). Emerging 2019 Novel Coronavirus (2019-nCoV pneumonia). Radiology, 295(1):210-7. 10.1148/radiol.2020200274

Suleyman G., Fadel R. A., Mallete K. M., Hamond C., Abdulla H., Entz A., Demertzis Z., Hanna Z., Failla A., Dagher C., Chaudhry Z., Vahia A., Lanfranco A. O., Ramesh M., ZervOS M. J., Alangaden G., Miller J., \& Brar I. (2020) Clinical characteristics and morbidity associated with coronavírus disease 2019 in a Serie of patients in metropolitan Detroit. JAMA Netw Open, 3(6):e2012270. 10.1001/jamanetworkopen.2020.12270

Wang D., Hu B., Hu C., Zhu F., Liu X., Zhang J., Wang B., Xiang H., Cheng Z., Xiong Y., Zhao Y., Li Y., Wang X., \& Peng Z. (2020). Clinical characteristics of 138 hospitalized patients with 2019 novel coronavirus-infected pneumonia in Wuhan. JAMA, 323(11):1061-9. 10.1001/jama.2020.1585

Wijaya I., Rizky A. \& Huang I. (2020). The Use of Therapeutic-Dose Anticoagulation and Its Effect on Mortality in Patients With COVID-19: A Systematic REVIEW. Clinical and Applied Thrombosis/Hemostasis, Sage journals, 26:1076029620960797. https://doi.org/10.1177/1076029620960797

World Health Organization (WHO). (2020). Corticosteroids for COVID-19. Geneva: WHO. https://www.who.int/publications/i/item/WHO- -2019-nCoVCorticosteroids-2020.1

World Health Organization (WHO). (2021). Covid-19. Geneva: WHO. https://www.who.int/publications/i/item/WHO- -2019-nCoV.

Xu X., Yu C., Qu, J., Zhang L., Jiang S., Huang D., Chen B., Zhang Z., Guan W., Ling Z., Jiang R., Hu T., Ding Y., Lin L., Gan Q., Luo L., Tang X., Liu J. (2020). Imaging and clinical features of patients with 2019 novel coronavirus SARS-CoV-2. European Journal of Nuclear Medicine and Molecular Imaging, 47:1275-1280. 10.1007/s00259-020-04735-9

Yang G., Zhang H. \& Yang Y (2020). Challenges and Countermeasures of Integrative Cancer Therapy in the Epidemic of COVID-19. Integr Cancer Ther, 19 $: 1534735420912811.10 .1177 / 1534735420912811$

Zhang L., Zhu F., Xie L., Wang C., Wang J., Chen R., Jia P., Guan H. Q., Peng L., Chen Y., Peng P., Zhang P., Chu Q., Shen Q., Wang Y., Xu S. Y., Zhao J. P., \& Zhou M. (2020). Clinical characteristics of COVID-19- infected cancer patients: a retrospective case study in three hospitals within Wuhan. Ann Oncol, 31(7):894-901]. 10.1016/j.annonc.2020.03.296

Zheng Y., Yang J., Gou X., Pu K., Chen Z., Guo Q., Ji R,, Wang H., Wang Y., \& Zhou Y. (2020). Prevalence of comorbidities and its effects in patients infected with SARS-CoV-2: a systematic review and meta-analysis. Int J Infect Dis, 94:91-5. 10.1016/j.ijid.2020.03.017

Zhou F., Yu T., Du R., Fan G., Liu Y., Liu Z., Xiang J., Wang Y., Song B., Gu X., Guan L., Wei Y., Li H., Wu X., Xu J., Tu S., Zhang Y., Chen H., \& Cao B. (2020). Clinical course and risk factors for mortality of adult inpatients with COVID-19 in Wuhan, China: a retrospective cohort study. Lancet, 395(10229):105462. $10.1016 / \mathrm{S} 0140-6736(20) 30566-3$ 